\title{
Les révolutions américaine et française et l'avancement de la démocratie
}

The American and French Revolutions and the Advance of Democracy

\section{Melvin Edelstein}

\section{(2) OpenEdition \\ 1 Journals}

\section{Édition électronique}

URL : https://journals.openedition.org/ahrf/871

DOI : 10.4000/ahrf.871

ISSN : 1952-403X

Éditeur :

Armand Colin, Société des études robespierristes

\section{Édition imprimée}

Date de publication : 1 décembre 2003

ISSN : 0003-4436

\section{Référence électronique}

Melvin Edelstein, «Les révolutions américaine et française et l'avancement de la démocratie », Annales historiques de la Révolution française [En ligne], 334 | octobre-décembre 2003, mis en ligne le 08 décembre 2009, consulté le 22 avril 2022. URL : http://journals.openedition.org/ahrf/871 ; DOI https://doi.org/10.4000/ahrf.871

Ce document a été généré automatiquement le 22 avril 2022.

Tous droits réservés 


\section{Les révolutions américaine et française et l'avancement de la démocratie}

The American and French Revolutions and the Advance of Democracy

Melvin Edelstein

1 Bien que les révolutions américaine et française soient considérées comme l'avènement de la démocratie moderne, certains Américains soutiennent la prééminence de la démocratie américaine sur le modèle français. Dans les années 60, les Américains ont même insisté sur le fait que les États-Unis étaient la démocratie la plus avancée dans la décennie 1790. Décrivant les États-Unis comme «la première nation nouvelle», les sociologues ont présenté la Révolution américaine comme un modèle pour les pays en voie de développement ${ }^{1}$.

Dans sa synthèse magistrale sur « la révolution démocratique » à la fin du XVIIIe siècle, tout en insistant sur l'élargissement de la démocratie en Amérique, R. R. Palmer maintient que les États-Unis étaient plus démocratiques que l'Europe durant la décennie révolutionnaire: "la vérité semble être que l'Amérique était beaucoup plus démocratique que l'Europe dans les années $1790{ }^{2}{ }^{2}$. La conclusion de Palmer étonne lorsqu'on sait sa connaissance de la Révolution française. L'idée que l'Amérique était la démocratie la plus avancée dans cette période est relayée par le politologue américain William Chambers: "la République américaine [note-t-il] a également produit le premier exemple de large suffrage dans le monde moderne. En dépit des limitations, bien plus d'hommes ont voté en Amérique qu'en Angleterre ou dans n'importe quel autre pays dans les années 1790 - ou, sur ce point, pendant les quelques décennies à venir $»^{3}$.

Plus récemment, Susan Dunn, professeur de littérature française, tout en comparant les deux «révolutions sœurs", insiste sur le fait que les conséquences de la Révolution américaine sont plus importantes et durables pour la démocratie. Cela s'explique par la différence des valeurs des révolutionnaires américains et français. Reprenant l'argument de Tocqueville, elle maintient que les Américains furent plus expérimentés 
dans la politique, tandis que les Français étaient des idéologues. Une discussion sur la culture politique des deux révolutions excède toutefois le cadre du présent article. Notre but est ici d'étudier comment les deux révolutions ont fait avancer la démocratie par le suffrage, les élections et la participation électorale ${ }^{4}$. Malgré l'affirmation que les États-Unis étaient le pays le plus démocratique des années 1790, jusqu'à quel point la Révolution américaine avait fait progresser la démocratie est le sujet de beaucoup de polémiques parmi les historiens américains. En 1926, J. F. Jameson a soutenu l'idée que la Révolution américaine avait mené l'Amérique dans la direction d'un "nivellement démocratique ${ }^{5}$. Dans les années 50 , cependant, les historiens ont insisté sur le conservatisme de la Révolution américaine. Le progrès de l'Amérique vers la démocratie politique et sociale s'est avéré plus limité que le pensait Jameson ${ }^{6}$. Plus récemment, les historiens américains ont souligné le radicalisme de la Révolution américaine. Elle a déclenché les forces qui au début du XIXe siècle avaient produit une vaste transformation sociale et politique. Gordon Wood affirme que «les Américains étaient devenus, bien que du jour au lendemain, les plus libéraux, les plus démocratiques, les plus entreprenants commercialement et les plus modernes du monde " 7 . La Révolution américaine, plus que n'importe quel autre événement, a fait de l'Amérique la nation la plus démocratique et la plus moderne au monde. Bien qu'il reconnaisse que la Révolution américaine faisait partie d'une expérience occidentale plus vaste, incluant la Révolution française, G. Wood repend l'argument que l'Amérique était le pays le plus démocratique au monde.

Pour évaluer jusqu'à quel point la France et les États-Unis étaient démocratiques dans les années 1790 , il est nécessaire de donner une définition de la démocratie ${ }^{8}$. La souveraineté populaire est le principe fondamental de la démocratie. Tocqueville a identifié la démocratie avec l'application la plus large de la souveraineté populaire ${ }^{9}$. L'article XXV de la Déclaration française des droits de l'homme et du citoyen du 24 juin 1793 précise : «La souveraineté réside dans le peuple ${ }^{10}$. Tout le pouvoir provient du peuple. Les fonctionnaires sont seulement des mandataires ; ils sont élus par le peuple, et responsables devant le peuple ${ }^{11}$. Des élections fréquentes et étendues sont essentielles à la démocratie. Puisque le gouvernement est établi par le peuple, les constitutions doivent être approuvées par le peuple.

Dans une démocratie, les citoyens obéissent seulement aux lois qu'ils se donnent euxmêmes. L'article VI de la Déclaration française des droits de l'homme et du citoyen de 1789 précise : « La loi est l'expression de la volonté générale. Tous les citoyens ont droit de concourir personnellement ou par leurs représentants à sa formation ». En fait, les citoyens font la loi à travers leurs représentants élus. Mais qui sont les «citoyens »? L'égalité des droits implique le suffrage universel. L'élimination de toutes les restrictions au droit de vote et à l'éligibilité est essentielle à la démocratie.

Puisqu'un moyen de mesurer la démocratie est l'application du principe électoral, nous comparerons jusqu'à quel point les postes étaient électifs en Amérique et en France. Selon R. R. Palmer, «l'avance de la démocratie politique dans la Révolution américaine était la plus évidente dans le choix très large des postes pour qui les électeurs pouvaient voter ${ }^{12}$. C'est généralement vrai, mais une analyse des constitutions fédérales et d'États dans les années 1790 montre que l'élection directe était limitée et que les membres du corps judiciaire ont toujours été nommés. Au niveau fédéral, seule la Chambre des représentants était élue directement. Les sénateurs étaient nommés par 
les législatures d'États, alors que le président et le vice-président étaient choisis par les électeurs dans chaque État. Tous les membres du corps judiciaire étaient nommés. Au niveau de l'État, les deux chambres de la législature étaient élues directement partout excepté pour le sénat au Maryland et au Kentucky de 1792 à 1799. Dans six des seize États existants en 1799 , le gouverneur était choisi par une assemblée électorale. Tous les membres du corps judiciaire étaient nommés, excepté le juge de la Cour suprême en Géorgie. Au niveau du comté, les juges de paix étaient nommés partout, alors que les shérifs étaient élus dans deux États seulement. Dans deux autres, les électeurs ont choisi deux candidats, puis le gouverneur en a nommé un.

Dans la France révolutionnaire, tous les législateurs, les administrateurs départementaux et de districts, les juges et le personnel des tribunaux, les juges de paix et leurs assesseurs, et les municipalités étaient élus; cependant, seules les deux dernières catégories étaient élues directement, tous les autres administrateurs, législateurs et juges l'étaient indirectement par les assemblées électorales. P. Gueniffey estime à plus d'un million le nombre de postes mis en place par la voie électorale ${ }^{13}$. Si on ne mesure la démocratie qu'au nombre de postes élus, elle a avancé plus loin en France qu'en Amérique.

Cependant, un autre moyen de mesurer la démocratie est la fréquence des élections. Les représentants américains sont élus tous les deux ans, les sénateurs sont choisis pour six ans, mais un tiers est remplacé tous les deux ans. Le président et le viceprésident sont élus pour quatre ans. Au niveau de l'État, les représentants étaient élus tous les ans, mais les membres de la chambre supérieure étaient élus dans les limites d'une à quatre années. Les gouverneurs étaient nommés pour une durée d'une à quatre années.

Les constitutions françaises de 1791, de 1793 et de 1795 ont établi des élections fréquentes et avec un renouvellement partiel. Cependant, la constitution de 1795 a établi un système général de "durée limitée. » Des électeurs et des fonctionnaires pouvaient être réélus seulement après un intervalle de plusieurs années. Si l'on en juge par la fréquence des élections, la rotation dans les postes, et la durée, la France était plus avancée que les États-Unis. II

12 Selon R. R. Palmer, ce qui distingue la Révolution américaine est le développement de la théorie du peuple en tant que pouvoir constituant. Les conventions constitutionnelles ont établi le gouvernement. La constitution du Massachussetts de 1780 a été rédigée par une convention élue par tous les hommes adultes et ratifiée par les électeurs. Mais beaucoup des constitutions des États, y compris celles du Tennessee (1796) et du Kentucky (1799), n'ont pas été soumises aux électeurs pour leur ratification. La constitution américaine de 1787 a été ratifiée indirectement par des conventions élues sur la base d'un droit de vote limité dans chaque État ${ }^{14}$. La Révolution française a dépassé l'Amérique dans deux voies: d'abord, toutes les constitutions, sauf celle de 1791, étaient ratifiées au suffrage direct et la ratification a été faite au suffrage masculin quasi universel ${ }^{15}$.

III

Une autre mesure de la démocratie consiste à observer jusqu'à quel point les élections sont directes ou indirectes. En Amérique, au niveau fédéral, la forme des élections n'était pas plus démocratique que dans le système français. Le président et le vice- 
président étaient nommés par un collège électoral. La Constitution a laissé le soin à la législature de chaque État de déterminer la méthode de choix des électeurs. En 1800, dans une grande majorité des États, les législatures ont choisi les électeurs. Les sénateurs étaient nommés par les législatures dans chaque État. L'élection directe était appliquée seulement aux membres de la Chambre des représentants. Puisque l'élection indirecte était établie aux États-Unis - comme en France - pour limiter la démocratie, l'Amérique n'était pas plus démocratique que la France.

La question de savoir jusqu'à quel point le suffrage était démocratique dans les deux pays est l'objet d'une polémique intense parmi les historiens des révolutions française et américaine ${ }^{16}$. Bien que les comparaisons soient difficiles parce que le mode de suffrage français a changé sept fois en une décennie, est-il vrai que la démocratie avait progressé davantage aux États-Unis? En Amérique, la détermination des critères pour le suffrage était laissée aux États. La Constitution stipule que «les électeurs dans chaque État auront satisfait aux conditions requises pour être électeurs de la chambre la plus nombreuse de la législature d'État ». Mais seulement deux des seize États, le Vermont et le Kentucky, ont eu le suffrage masculin universel en 1799. Cependant, parce qu'il n'y avait aucun critère pécuniaire ou religieux pour les postes fédéraux, l'Amérique avait fait progresser la démocratie.

16 Aux États-Unis, où la réforme du suffrage avait lieu État par État, l'avance de la démocratie a suivi les précédents établis par la Révolution française : une transition de l'héritage anglais de propriétaire possédant le droit de vote à un régime censitaire, jusqu'à ce que le suffrage universel ait été finalement réalisé. Le suffrage masculin blanc universel a été réalisé dans la période qui suit la Révolution française et avant la Guerre de Sécession. Cependant, l'Amérique a été plus en avance que la France en éliminant les critères religieux pour des postes. Cependant, les conditions élevées de propriété pour être éligible aux charges ont contrecarré l'avancement de la démocratie.

17 La suppression des critères de propriété pour le droit de vote a été citée comme une évidence pour montrer l'avancement de la démocratie aux États-Unis. En 1796, sept des seize États alors dans l'union n'avaient aucune condition de propriété pour être électeur dans le choix de la Chambre législative inférieure ${ }^{17}$. D'autre part, dans la « Northwest Ordinance » de 1787, le congrès a exigé une propriété foncière perpétuelle de 50 acres (approximativement 25 hectares) pour être électeur des législatures dans les nouveaux États. La condition était si restrictive qu'elle a dû être abandonnée ${ }^{18}$.

En Amérique, on a accordé le suffrage aux hommes ayant un état permanent dans la société, comme en France en $1789^{19}$. D'une façon générale, le droit de vote a été accordé aux hommes blancs âgés d'au moins vingt et un ans, résidant depuis six mois à deux ans, satisfaisant certaines conditions pécuniaires. Vers 1799, seulement deux États, le Kentucky et le Vermont avaient supprimé toutes les conditions pécuniaires pour accorder le suffrage. Quatre États, le Delaware, la Géorgie, le New Hampshire et la Pennsylvanie avaient un régime censitaire. Dix États ont exigé une propriété quelconque pour ce droit. En Caroline du Sud, le suffrage a été accordé aux propriétaires fonciers ou aux contribuables. Au Tennessee, les propriétaires fonciers ou les hommes libres résidant dans n'importe quel comté pendant six mois pouvaient voter. L'État de New York et la Caroline du Nord ont exigé une condition plus élevée pour la Chambre supérieure.

19 Malgré ces restrictions, le corps électoral était-il aussi large que R. R. Palmer et W. Chambers le prétendent? Bien que les historiens américains soient en désaccord au 
sujet de l'impact de ces limitations du droit de vote, même dans la période coloniale, $50 \%$ à $90 \%$ des hommes adultes pouvaient satisfaire aux exigences du suffrage ${ }^{20}$. Dans ces États qui ont adapté un régime censitaire, le corps électoral était large, ce qui était dû à l'incidence très élevée de l'imposition ${ }^{21}$. Arguant du fait que la propriété foncière était répandue et l'offre économique abondante, Robert Brown maintient que $90 \%$ des hommes adultes avaient le droit de vote dans le Massachussetts. D'autre part, les estimations pour la Virginie varient entre un tiers d'hommes adultes et jusqu'à $85 \%^{22}$.

Considérant que le corps électoral était très large, les États-Unis étaient-ils plus démocratiques que la France révolutionnaire? Les historiens affirment que les élections aux États généraux furent parmi les plus démocratiques de la Révolution. Seuls les électeurs du Tiers État devaient être inscrits sur le rôle de taille et il n'y avait aucune condition pour l'éligibilité ${ }^{23}$. D'autre part, le vote par ordres et corporations et le vote indirect pour les députés, qui étaient seulement les mandataires de leurs communautés, invalidaient le dispositif démocratique.

21 Le suffrage établi en décembre 1789 a traduit la philosophie selon laquelle seuls ceux ayant une volonté indépendante ont été autorisés à voter. Ceci a été mesuré par l'imposition. Les citoyens étaient divisés en quatre catégories $: 1^{*}$ citoyens passifs qui ont eu des droits civiques seulement ; $2^{*}$ citoyens actifs qui ont eu seulement le droit de vote ; $3^{*}$ éligibles, qui pouvaient être élus ; $4^{*}$ ceux qui pouvaient être élus député. Toutes les élections étaient indirectes, exceptées celles pour les municipalités et les juges de paix. Cependant, le système électoral était fondé sur un large corps électoral pour donner une légitimité démocratique à des élus choisis par des assemblées électorales plus exclusives ${ }^{24}$.

Les dispositions restrictives au droit de suffrage ont-elles créé une classe «bourgeoise " privilégiée ou " une aristocratie de richesse »? R. R. Palmer affirme que presque $70 \%$ des hommes adultes sont citoyens actifs ; que les trois quarts d'entre eux, et environ la moitié des hommes adultes étaient éligibles, et environ $1 \%$ des hommes adultes et $10 \%$ des citoyens actifs pouvaient être députés ${ }^{25}$.

Des études récentes confirment les conclusions de Palmer ${ }^{26}$. Nous estimons que $60 \%$ à $70 \%$ des hommes adultes sont citoyens actifs, mais seulement $60 \%$ de ces derniers et $40 \%$ des hommes adultes étaient éligibles. Comparé à l'Amérique, le suffrage avait une base très large et les critères requis pour l'éligibilité à des postes locaux n'étaient pas aussi exclusifs que les historiens le prétendent. En plus de créer un assez large suffrage, les révolutionnaires ont éliminé la religion comme une condition pour avoir le droit de vote et être élu.

24 La Constitution de 1791, qui n'a jamais été appliquée, était le résultat d'une réaction anti-démocratique. Tout en laissant la citoyenneté active intacte, elle a limité de manière significative les conditions d'éligibilité aux propriétaires, aux locataires, aux fermiers et aux métayers. Le marc d'argent a été supprimé ; dorénavant n'importe quel citoyen actif pouvait être élu député.

En raison de l'insurrection du 10 août 1792, la France est devenue une République, comme les États-Unis. La suppression de la distinction entre citoyens actifs et citoyens passifs a mené les historiens à conclure que le suffrage masculin universel avait été établi ${ }^{27}$. Ceci est cependant exagéré parce que les votants à la Convention nationale devaient vivre d'un revenu ou du produit de leur travail. Les domestiques étaient exclus, de même que les femmes, les non-résidents, les travailleurs ambulants, les chômeurs, et les enfants mineurs. L'abaissement de l'âge du vote à vingt et un ans a 
rendu la France semblable aux États-Unis. Bien que des élections indirectes aient été maintenues, toutes les conditions pécuniaires pour l'éligibilité étaient supprimées. Le paiement d'une indemnité aux électeurs était une innovation importante. La suppression de n'importe quelle condition de cens pour le droit de vote et d'éligibilité a fait de la France le pays le plus démocratique.

Bien qu'elle n'ait jamais été appliquée, la Constitution de 1793 a fait de la France la première république démocratique dans l'histoire moderne, un fait souvent ignoré par les Américains. Les domestiques n'étaient plus exclus. Le résultat était un accroissement très important du nombre des votants ${ }^{28}$. Pour la première et seule fois pendant la Révolution, des députés devaient être élus directement - comme les membres du Congrès américain. Les lois devaient être soumises à une sorte de référendum. La Constitution de 1793 était la première constitution française soumise à la ratification populaire.

Après la suspension des élections par le gouvernement révolutionnaire, la Constitution de 1795 a rétabli une république libérale avec la première législature bicamérale en France. Elle était rédigée pour prévenir à la fois le retour de la démocratie et de la dictature. Le droit de vote était semblable à celui établi par la Constitution de 1791. Cependant, le suffrage était plus large parce que l'âge du vote avait été abaissé de vingtcinq à vingt et un ans et les citoyens devaient payer un impôt direct. Mais les soldats qui avaient combattu dans une ou plusieurs campagnes pour établir la république étaient exempts de la condition d'imposition. En payant volontairement un impôt égal à la valeur de trois journées de travail, quiconque pouvait devenir votant.

Malgré tout, la Constitution a eu plusieurs dispositions restrictives. À partir de l'an XII (1805), il fallait savoir lire, écrire et exercer une profession mécanique ou agricole. Les domestiques étaient exclus du vote. Les lois suivantes écartèrent d'autres groupes. La loi du 3 brumaire an IV (15 octobre 1795) exclut: $1^{*}$ les prêtres réfractaires; $2^{*}$ certaines catégories d'émigrés ; $3^{*}$ les parents d'émigrés. Reflétant l'idée que la France devait être dirigée par les propriétaires, les conditions pour être électeur étaient très restrictives. Comme en 1791, il fallait être propriétaire, locataire, fermier, ou métayer. Cependant, il n'y avait aucune condition pécuniaire pour être député.

Bien que Jacques Godechot maintienne que la Constitution de 1795 fût peu démocratique, Malcolm Crook argue du fait que le corps électoral était supérieur à celui de $1791{ }^{29}$. Alors qu'en 1791, environ $16 \%$ de la population et 60 à $70 \%$ des hommes adultes de vingt-cinq ans et plus bénéficiaient du droit de vote, l'estimation pour la période du Directoire varie entre $18 \%$ et $22 \%$ de la population et près de $80 \%$ des hommes adultes âgés de vingt et un ans et plus. Cependant, les conditions d'éligibilité furent tellement restrictives que $10 \%$ seulement du corps électoral fut capable d'être élu ${ }^{30}$.

30 La Constitution de 1799 a restauré le suffrage masculin universel à l'exception des domestiques. Mais, si ce suffrage était bien démocratique, on a seulement permis aux électeurs de créer des listes de notabilités dans lesquelles les administrateurs étaient choisis. De plus, il n'y avait aucune condition pécuniaire pour être élu. À l'époque napoléonienne, les élections révolutionnaires étaient supprimées, la longue décennie de l'apprentissage de la démocratie en France était close.

31 Même si on peut dire que l'Amérique coloniale a eu le plus large électorat dans le monde ${ }^{31}$, ce n'était plus vrai dans les années 1790 . Si l'on considère que 60 à $70 \%$ des hommes adultes avaient le droit de vote en France en 1789-1792, que le corps électoral 
était encore plus important dans la période du Directoire, et que la France a établi le suffrage quasi universel en 1792 et le suffrage masculin universel en 1793, la France était plus démocratique que l'Amérique dans les années 1790 .

\section{qu'on ait permis aux contribuables de voter pour la Chambre basse en Carreline du} qu'on ait permis aux contribuables de voter pour la Chambre basse en Caroline du Nord, une propriété foncière perpétuelle de 100 acres (approximativement 40 hectares) était exigée pour les représentants. Les sénateurs, qui étaient choisis par des électeurs possédant 50 acres de terre (approximativement 20 hectares), devaient posséder 300 acres (approximativement 120 hectares). Le gouverneur, désigné par la législature, devait avoir une propriété foncière perpétuelle d'une valeur de 1000 livres et être protestant ${ }^{32}$. En Caroline du Sud, où les possédants d'une propriété foncière perpétuelle de 50 acres (approximativement 20 hectares) ou d'une propriété en ville, ou les contribuables ayant payé 3 shillings sterling pouvaient voter pour les deux chambres, les représentants devaient avoir une propriété foncière perpétuelle de 500 acres (approximativement 200 hectares) et dix nègres ou posséder une propriété d'une valeur de 150 pounds sterling. Les sénateurs devaient détenir une propriété foncière perpétuelle d'une valeur de 300 pounds sterling. Le gouverneur et le lieutenantgouverneur, tous les deux choisis par la législature, devaient disposer d'un domaine d'une valeur de 1500 pounds sterling. Seuls les hommes libres étaient autorisés à voter et à être élus aux postes d'État ${ }^{33}$.

Le Maryland et le Kentucky ont établi les assemblées électorales pour élire les sénateurs. Bien que pour ce dernier aucun critère pécuniaire n'ait été requis, le Maryland eut des conditions restrictives de propriété pour les électeurs et les sénateurs.

La Nouvelle Angleterre a également eu un système de conditions de propriété pour les postes d'État. En se basant sur la valeur du dollar en 1792 estimée par R. R. Palmer, même un membre de la Chambre des représentants du Massachussetts devait posséder une propriété dont la valeur était plus élevée que le montant du cens exigé des députés français en 1789-1791 ou la propriété demandée aux électeurs dans les Constitutions de 1791 et de $1795^{34}$.

$\mathrm{Vu}$ ces conditions restrictives de propriété pour les législatures d'état et les gouverneurs, la démocratie n'avait pas progressé plus loin en Amérique. Bien que la 
France ait eu des élections indirectes pour la plupart des postes, les critères de cens ou de propriété pour l'égibilité semblent être inférieurs à ceux en usage en Amérique. forte peut être trouvée en 1790 , quand $50 \%$ au moins des citoyens actifs votaient aux élections administratives; un pourcentage encore plus élevé de ruraux avait participé auparavant aux élections municipales. Ceci signifie que plus de deux millions de citoyens ont voté, un chiffre bien plus élevé que le vote en Amérique. Aux élections législatives de juin 1791, le vote a chuté précipitamment à au moins $23-24 \%$, soit approximativement un million et plus de votants. Le vote aux élections à la Convention nationale est estimé à seulement $15 \%$ à $20 \%$, mais d'un corps électoral plus élargi. Environ 1,8 million de citoyens ont voté au plébiscite constitutionnel de 1793, soit à peu près $30 \%$ du corps électoral. De 1795 à 1799, la participation est tombée à environ $20 \%$, plongeant à environ $10 \%$ en $17999^{41}$. 

élevée qu'en Amérique, le vote plus faible en 1791, 1792, et de 1795 à 1799, n'était pas nécessairement inférieur au vote en Amérique. Si l'on considère que la France n'avait pas développé les partis politiques, les campagnes électorales, ou les candidatures proclamées, alors que tout cela existait aux États-Unis, la faible participation française est compréhensible ${ }^{42}$. Quant aux niveaux élevés atteints par l'Amérique pour la participation aux élections présidentielles en 1828 et après 1840 , ils étaient le résultat des candidats de l'opposition et le fruit de l'organisation des partis pour mobiliser les électeurs ${ }^{43}$.

\section{VI}

Que les États-Unis soient un des pays les plus démocratiques est indéniablement vrai. Mais l'affirmation que l'Amérique était la démocratie la plus avancée dans les années 1790 est une exagération. La comparaison de la République américaine à son début avec la France révolutionnaire montre que, dans beaucoup de faits, la démocratie avait progressé plus loin en France. Ceci semble être ignoré par les Américains.

Dans les années 1790, les États-Unis et la France ont développé deux modèles différents de démocratie. L'Amérique a développé un pluralisme, un bipartisme, une politique de groupes d'intérêts. La France n'a pas embrassé ce modèle. Les révolutionnaires ont rejeté les candidatures proclamées, les partis politiques et les campagnes électorales. Solliciter les suffrages, briguer des postes étaient les moyens certains d'attirer le discrédit. D'ailleurs, l'identification de la démocratie avec la dictature révolutionnaire et la Terreur donne de la contribution française à la démocratie une certaine ambivalence ${ }^{44}$. Même aujourd'hui, la France et les États-Unis offrent deux modèles concurrents de démocratie.

\section{NOTES}

1. Seymour Martin LIPSET, The First New Nation, New York, 1979. Ce livre a paru en 1963.

2. R. R. PALMER, The Age of the Democratic Revolution, 2 vol., Princeton, 1959-1964, I, p. 234. «The truth seems to be that America was a good deal more democratic than Europe in the 1790's».

3. William NESBIT CHAMBERS, Political Parties in a New Nation: The American Experience, 1776-1809, New York, 1963, p. 124. "The American republic also produced the earliest instance of extended suffrage in the modern world. Despite limitations, far more men voted in America than in England or in any other country in the 1790's-or, for that matter, for some decades to come».

4. Susan DuNN, Sister Revolutions : French Lightning, American Light, New York, 1999.

5. J. Franklin JAMESON, The American Revolution Considered as a Social Movement, Princeton, 1926.

6. Frederick TOLLES, "The American Revolution Considered as a Social Revolution: a Reevaluation», American Historical Review, LX, October, 1954, p. 1-12; Elisha DougLASS, Rebels and Democrats: The Struggle for Equal Political Rights and Majority Rule during the American Revolution, Chapel Hill, 1955.

7. Gordon wood, The Radicalism of the American Revolution, New York, 1993, pp. 6-7. 
8. Pour les définitions de la démocratie, voir Philippe REYNAUD, «Démocratie» dans le Dictionnaire critique de la Révolution française, Paris, 1988. Voir également les articles de Maurice GENTY sur «Démocratie directe» et «Démocratie représentative» dans le Dictionnaire historique de la Révolution française, sous la direction de Jean-René SURATTEAU et François GENDRON, Paris, 1989, pp. 340-342.

9. Alexis De tocqueville, De la Démocratie en Amérique, 2 vol., Paris, 1835-40, I, chapitre 4.

10. Pour les textes des constitutions françaises, voir Voter, élire pendant la Révolution française 1789-1799: Guide pour la recherche, rédigé par Serge ABERDAM, Serge BIANCHI, Robert DEMEUDE, Émile DUCOUDRAY, Bernard GAINOT, Maurice GENTY et Claudine WOLIKOW, Paris, 1999.

11. Voir Article IV de la constitution de l'État de Pennsylvanie en 1776. Francis N. THORPE, Federal and State Constitutions, Colonial Charters and Other Organic Laws of the... United States of America, 7 vol., Washington, D.C., 1909, V, p. 3082.

12. R. R. PALMER, op. cit., I, p. 233.

13. Patrice GUENIFFEY, Le nombre et la raison: La Révolution française et les élections, Paris, 1993.

14. R. R. PALMER, op. cit., I, pp. 213-235.

15. Serge ABERDAM, "L'élargissement du droit de vote entre 1792 et 1795 au travers du dénombrement du Comité de Division et des votes populaires sur les constitutions de 1793 et 1795», Thèse de Doctorat, Université Paris I-Sorbonne, U.F.R. d'histoire, 2001.

16. R. R. PALMER, op. cit., I, pp. 522-528.

17. Id., I, p. 233.

18. Chilton WiLliamson, American Suffrage from Property to Democracy, 1760-1860, Princeton, 1960, pp. 117-118.

19. Voir la déclaration des droits de l'État de Virginie en 1776 dans F. THORPE, op. cit., VII, p. 3813.

20. Robert DINKIN, Voting in Provincial America : A Study of Elections in the Thirteen Colonies, 1689-1776, Westport, Conn., 1977, pp. 46-49 ; C. WiLliamson, op. cit., p. 139.

21. Id.., pp. 135-136.

22. Robert BROWN, Middle-Class Democracy and the Revolution in Massachussetts, 1691-1780, Ithaca, 1955 ; Charles SYDNOR, American Revolutionaries in the Making: Political Practices in Washington's Virginia, New York, 1962, p. 38 ; Robert and Katherine BROWN, Virginia, 1705-1786: Democracy or Aristocracy?, East Lansing, 1964.

23. R. R. PALMER, op. cit., I, p. 476 ; William DOYLE, The Oxford History of the French Revolution, Oxford, 1989, p. 97 ; François FURET, «La monarchie et le règlement électoral de 1789», dans The Political Culture of the Old Regime, éd. Keith BAKER, New York, 1988, pp. 375-386.

24. Voir les lois du 14 et 22 décembre 1789. Pour voter, il fallait être : (1) un citoyen ; (2) âgé d'au moins 25 ans ; (3) résidant dans une ville ou un canton depuis au moins une année ; (4) et payant un impôt

égal à la valeur locale de trois journées de travail. Les femmes, les serviteurs à gages, les enfants dépendants, les accusés de crime, les débiteurs insolvables, et les faillis étaient exclus du droit de vote. Les électeurs ont également dû prêter un serment civique et, après juin 1790, pour être inscrits dans les registres de la Garde nationale. Pour être éligible, il fallait payer un impôt direct égal de la valeur locale de dix journées de travail. Les députés devaient payer un impôt égal à la valeur d'un marc d'argent (54 livres) et posséder une propriété. L'Assemblée nationale a fixé la valeur d'une journée de travail entre 10 et 20 sous. Il fallait payer 1,5 à 3 livres d'impôts pour avoir le droit de vote et de 5 à 10 livres pour être éligible.

25. R. R. PALMER, op. cit., I, pp. 522-528.

26. P. GUENIFFEY, op. cit., pp. 31-58; Malcolm CROOK, Elections in the French Revolution: An Apprenticeship in Democracy, 1789-1799, Cambridge, 1996, pp. 30-53 ; Melvin EDELSTEIN, «Electoral Behavior During the Constitutional Monarchy (1790-1791) : A «Community Interpretation» dans The French Revolution and the Meaning of Citizenship, éd. Renée WALDINGER, Philip DAWson, et Isser 
WOLOCH, Westport, Conn., 1993, p. 105 ; Melvin EDELSTEIN, «Participation et sociologie électorales des Landes en 1790», A.H.R.F., n³16, avril-juin 1999, pp. 306-310.

27. W. DOYLE, op. cit., p. 193 ; Melvin EDELSTEIN, «L'Établissement de la République en Côte-d'Or: étude électorale et politique», dans Révolution et République : L'Exception française, sous la direction de Michel Vovelle, Paris, 1994, pp. 226-227.

28. S. ABERDAM, op. cit.

29. Jacques GODECHOT, Les Institutions de la France sous la Révolution française et l'Empire, Paris, 1951, p. 399 ; M. CROOK, op. cit., p. 118.

30. Id.

31. R. DINKIN, op. cit., p. 49.

32. F. THORPE, op. cit., V, pp. 2787-2794.

33. Id., VI, pp. 3528-3625.

34. R. R. PALMER, op. cit., I, p. 527.

35. Id., I, p. 227 n.

36. Id., I, p. 231.

37. R. R. PALMER, The World of the French Revolution, New York, 1972, p. 69.

38. C. SYDNOR, op. cit., p. 121.

39. Richard мссовміск, The History of Voting in New Jersey, New Brunswick, 1953, p. 121.

40. Jack POLE, «Suffrage and Representation in Massachussetts : A Statistical Note», William and Mary Quarterly, XIV (1957), 586-587. Pour les statistiques électorales, voir Jack POLE, Political Representation in England and the Origins of the American Republic, New York, 1966, pp. 543-564.

41. Melvin EDELSTEIN, «Le comportement électoral sous la monarchie constitutionnelle (1790-1791) : Une interprétation "Communautaire" ", A.H.R.F., n 301 (1995), pp. 361-398 ; Melvin EDELSTEIN, «La participation électorale des français (1789-1870)», Revue d'histoire moderne et contemporaine, XL-4 (1993), 631 ; M. CROOK, op. cit. ; P. GUENIFFEY, op. cit., p. 186.

42. Melvin EDELSTEIN, «La culture électorale française, de l'époque révolutionnaire à l'époque napoléonienne», dans La plume et le sabre: Hommages offerts à Jean-Paul Bertaud, Paris, 2000, pp. 489-497. Malcolm сRоок, «Le candidat imaginaire, ou l'offre et le choix dans les élections de la Révolution française», A.H.R.F., $\mathrm{n}^{\circ} 321$ (2000), pp. 91-92.

43. Richard McCoRMick, « New Perspectives on Jacksonian Politics», American Historical Review, XLV (1960), pp. 288-301.

44. Pour le développement de la culture politique française, voir Philippe TANCHOUX, «Procédures et pratiques électorales en France de la fin de l'Ancien Régime à la veille de la Première guerre mondiale», Thèse pour le Doctorat, Université d'Orléans, 2000.

\section{RÉSUMÉS}

Bien que les révolutions américaine et française soient considérées comme l'avènement de la démocratie moderne, certains Américains soutiennent la prééminence de la démocratie américaine sur le modèle français. Dans les années 60, les Américains ont même insisté sur le fait que les États-Unis étaient la démocratie la plus avancée dans la décennie 1790 jusqu'à 1799. Le but de cet article est de comparer comment les deux révolutions ont fait avancer la démocratie. La comparaison de la République américaine à son début avec la France révolutionnaire montre que dans beaucoup de faits la démocratie avait progressé plus loin en France. 
Although the American and French revolutions are considered as the rise of modern democracy, certain Americans claim that American democracy was superior to the French model. In the 1960's, Americans argued that the United States was the most advanced democracy in the decade 1790-1799. The aim of this article is to compare how the two revolutions advanced democracy. A comparison of the fledgling American republic with revolutionary France shows that in many ways democracy had advanced further in France.

\section{INDEX}

Mots-clés : démocratie, suffrage, éligibilité, élections, vote, Suffrage 